\title{
Hydrolyzed Formula With Reduced Protein Content Supports Adequate Growth: A Randomized Controlled Noninferiority Trial
}

\author{
${ }^{*}$ Birgit Ahrens, ${ }^{\top}$ Christian Hellmuth, ${ }^{\dagger}$ Nadja Haiden, ${ }^{\ddagger}$ Dirk Olbertz, ${ }^{\S}$ Eckard Hamelmann, \\ "Milica Vusurovic, "Manja Fleddermann, " Robert Roehle, ${ }^{* *}$ Anette Knoll, \\ "Berthold Koletzko, *Ulrich Wahn, and * Kirsten Beyer
}

\begin{abstract}
Objective: A high protein content of nonhydrolyzed infant formula exceeding metabolic requirements can induce rapid weight gain and obesity. Hydrolyzed formula with too low protein (LP) content may result in inadequate growth. The aim of this study was to investigate noninferiority of partial and extensively hydrolyzed formulas (pHF, eHF) with lower hydrolyzed protein content than conventionally, regularly used formulas, with or without synbiotics for normal growth of healthy term infants.

Methods: In an European multi-center, parallel, prospective, controlled, double-blind trial, 402 formula-fed infants were randomly assigned to four groups: LP-formulas $(1.9 \mathrm{~g}$ protein $/ 100 \mathrm{kcal})$ as $\mathrm{pHF}$ with or without synbiotics, LP-eHF formula with synbiotics, or regular protein eHF $(2.3 \mathrm{~g}$ protein $/ 100 \mathrm{kcal})$. One hundred and one breast-fed infants served as observational reference group. As primary endpoint, noninferiority of daily weight gain during the first 4 months of life was investigated comparing the LP-group to a regular protein eHF group.

Results: A comparison of daily weight gain in infants receiving LPpHF $(2.15 \mathrm{~g} /$ day $\mathrm{CI}-0.18$ to inf.) with infants receiving regular protein eHF showed noninferior weight gain $(-3.5 \mathrm{~g} /$ day margin; per protocol [PP] population). Noninferiority was also confirmed for the other tested LP formulas. Likewise, analysis of metabolic parameters and plasma amino acid concentrations demonstrated a safe and balanced nutritional composition. Energetic efficiency for growth (weight) was slightly higher in LPeHF and synbiotics compared with LPpHF and synbiotics.

Conclusions: All tested hydrolyzed LP formulas allowed normal weight gain without being inferior to regular protein eHF in the first 4 months of life. This trial was registered at clinicaltrials.gov, NCT01143233.
\end{abstract}

Key Words: growth development, hydrolysates, protein content

(JPGN 2018;66: 822-830)

Received February 6, 2017; accepted November 15, 2017.

From the *Department of Pediatric Pneumology and Immunology, Charité Universitätsmedizin Berlin, Berlin, Germany, the †Division of Neonatology, Pediatric Intensive Care Medicine and Neuropediatrics, Department of Pediatrics and Adolescent medicine, Medical University of Vienna, Austria, the $\ddagger$ Department of Neonatology, Klinikum Südstadt Rostock, the §Children's Center Bethel, Bielefeld, and Allergy Center, Ruhr University, Bochum, Germany, the $\|$ Department of Neonatology, Clinical Hospital Center Belgrade, Srbija, the ${ }^{\text {Ludwig-Maximilians-Univer- }}$ sität München, Div. of Metabolic and Nutritional Medicine, Dr. von Hauner Children's Hospital, University of Munich Medical Center, the \#Coordination Center of Clinical Studies, KKS, Charité Universitätsmedizin Berlin, Berlin, and the **AK Statistics, Kreppe 2, Pfaffenhofen, Germany.

\section{What Is Known}

- The protein content of infant formula has been positively associated with rapid weight gain and obesity.

- Hydrolyzed formulas are used for allergy prevention.

- Data on the effect of hydrolyzed formulas with reduced hydrolyzed protein content are rare.

\section{What Is New}

- Hydrolyzed formulas with reduced protein content are suited to ensure normal growth during the first 4 months of life.

- Length $z$ scores, weight and head circumference $z$ scores were similar to World Health Organization Standards in all groups.

- Extensively hydrolyzed infant formula showed a higher energetic efficiency compared with partially hydrolyzed formula.

E arly childhood diet and rapid early growth have been identified as important predictors of long-term health through to adulthood (1-3). An inverse relationship has been documented between obesity development and breast-feeding (BF) duration suggesting the latter's protective role (4). Formula-fed term infants have been found to show greater body weight gain compared with breast-fed babies (5). Particularly the higher protein intake of formula milk has been suggested as an important stimulus for the accelerated infant growth via increased secretion of insulin-like growth factor I $(5,6)$.

Address correspondence and reprint requests to Kirsten Beyer, MD, Department of Pediatric Pneumology and Immunology, Charité -Universitätsmedizin Berlin, Augustenburger Platz 1, 13353 Berlin, Germany (e-mail: kirsten.beyer@charite.de).

Supplemental digital content is available for this article. Direct URL citations appear in the printed text, and links to the digital files are provided in the HTML text of this article on the journal's Web site (www.jpgn.org).

www.clinicaltrials.gov registration number: NCT01143233.

This study was funded by HiPP GmbH \& Co. Vertrieb KG, Pfaffenhofen, Germany.

The extensively hydrolyzed protein used for $\mathrm{eHF}$ and $\mathrm{LPeHF}+\mathrm{PP}$ group was Peptigen IF-3080 (Arla Foods Ingredients). 
In a systematic review on the effects of infant protein intake and growth, 12 randomized controlled trials (RCTs) were analyzed, and wherever possible, a meta-analysis was performed (7). The authors conclude that "the current evidence is insufficient for assessing the effects of reducing the protein concentration in infant formulas on long-term outcomes, but, if confirmed, this could be a promising intervention for reducing the risk of overweight and obesity in children."

There is hardly any data available regarding the safety of hydrolyzed formula with lower protein (LP) content (8). Moreover, authorities do not accept the transfer of study results from one hydrolysate to another (9). Nevertheless, in line with current European and American prevention guidelines for allergic diseases, infants at high-risk can be recommended a hypoallergenic (hydrolyzed) formula (HA) with a documented preventive effect for the first 4 months, if BF is impossible (10-14).

The goal of this study was to investigate safety and suitability of infant formulas with LP content using a noninferiority design. On the basis of the only other safety study on hydrolyzed proteins, which had already proven safety by the EFSA committee at the start of our study, the acceptable noninferiority margin for evaluating the daily weight gain $(-3.5 \mathrm{~g} /$ day $)$ was derived (8). As primary endpoint, noninferiority of daily weight gain was investigated comparing the LP group (LPpHF $1.9 \mathrm{~g}$ protein $/ 100 \mathrm{kcal}$, allowed by Directive 2006/141/EC) to a regular protein eHF group (2.3 g protein $/ 100 \mathrm{kcal})$.

In addition to aspects of the protein content, other ingredients of human milk like oligosaccharides as well as probiotic bacteria have been discussed as contributing to health benefits of breast-fed infants (15-17), probiotics and prebiotics (synbiotics) were added.

Combining these aspects, the aim of this study was to investigate noninferiority for normal growth of healthy term infants of partial and extensively hydrolyzed formulas (pHF, eHF) with lower hydrolyzed protein content than regularly used conventional formulas, with or without synbiotics.

\section{PATIENTS AND METHODS}

\section{Trial Design}

The study was a parallel, prospective, randomized, controlled, double-blind, intervention trial. Noninferiority with regard to growth was tested comparing three modified intervention formulas to an established formula. The study was conducted according to the principles and rules laid down in the Declaration of Helsinki and its subsequent amendments, also in accordance with the International Conference on Harmonization Guidelines on Good Clinical
Practice, and following the recommendations stated in the Consolidated Standards of Reporting Trials (CONSORT) guidelines (18). Procedures were approved by the ethics committees of all study centers. Written informed parental consent was obtained. This trial was registered at clinicaltrials.gov, NCT01143233. (More details are available in the Supplementary Appendix, Supplemental Digital Content, http://links.lww.com/MPG/B201).

All infant formulas are in accordance with relevant EU directives (Directive 2006/141/EC) (19). Formulas were wheybased, either extensively or partially hydrolyzed (eHF or pHF) and differed from the regular formula in the amount of hydrolyzed cow milk-based protein: LP formula (1.9 g protein $/ 100 \mathrm{kcal})$ as $\mathrm{pHF}$ with synbiotics, without synbiotics, eHF-LP-formula with synbiotics, or regular protein eHF (2.3 g protein $/ 100 \mathrm{kcal}$; Table 1$)$. Currently, no formula is available on the market.

For the synbiotics, Lactobacillus fermentum CECT5716 was used as probiotic (20) and galacto-oligosaccharides (GOS) as prebiotic. Individual amino acids (AAs) were added according to Annex V of 2006/141/EC (Table 1).

\section{Primary and Secondary Outcome Assessment}

The study was designed as a safety study (up to 4 months of age) with an additional follow-up visit at 12 months of age. The primary outcome analysis was based on growth assessed up to 4 months of age, which is recommended by Scientific Committee on Food (SCF) report 2003. The primary outcome of the study was the average daily weight gain per day in grams (g/day) between Visit 1 ( $28 \pm 3$ days of life) and Visit 4 (112 \pm 3 days of life) to estimate proper growth of infants fed the lower protein hydrolyzed infant formula. Secondary outcomes included measurements of: body length and head circumference as well as the analysis of metabolic parameters. To describe adverse events (AE), the following subgroups were regarded with special interest: atopic dermatitis, fever episodes, and infections.

\section{Study Population}

Included were healthy term newborns $<28$ days of life with a gestational age of $\geq 37$ weeks and a birth weight between 2500 and $4500 \mathrm{~g}$. During recruitment procedures, BF was promoted. Exclusion criteria were any severe acquired or congenital illness in infants or mothers that could potentially affect normal growth, (ongoing) antibiotic therapy, a regular intake of supplementary synbiotics by the child and/or BF mother, and the participation in another clinical trial.
B.A. received a travel reimbursement and honorarium from Allergopharma for active participation in a scientific seminar. N.H. has received speaker's honoraria from Nestle, Milupa, Hipp, Novolac, and Baxter, is running studies for Nestle, Hipp, and Winicker Norimed and is currently receiving a grant (No: 15378) from the Austrian National Bank "Jubilee Fund." E.H. received a travel reimbursement and honorarium from Nutricia and Milupa for his active participation in scientific seminars. Analyses of energetic efficacy of infant formulae by M.F. were performed within her research activity at the Dr. von Hauner Children's Hospital Munich. M.F. is now an employee of HiPP GmbH \& Co. Vertrieb KG. A.K. has received honoraria from HiPP $\mathrm{GmbH} \&$ Co. Vertrieb KG. B.K. is a member of the National Breastfeeding Committee and declares bias towards breastfeeding. The LudwigMaximilians-University (LMU) of Munich Medical Centre and its employees B.K. and C.H. had scientific and educational collaboration with manufacturers of nutritional products for infants and children, primarily as part of research collaboration funded by the European Commission, the European Research Council and also with German government funding. The work performed at LMU and reported in this manuscript has been financially supported in part by Hipp. B.K. does not report a conflict of interest, which would represent "a set of circumstances that creates a risk that professional judgment or actions regarding a primary interest will be unduly influenced by a secondary interest," as defined by the US Institute of Medicine. U.W. received Speaker's fees: ALK, Allergopharma, Stallergenes, Nutricia, Nestle, FAES Pharma; Consultation fees: ALK, Allergopharma, Stallergenes, Novartis, Danone, Hipp, MEDA, Biomay. K.B. has no direct conflict of interest. However, she reports grants and personal fees outside the submitted work from the European Union, German Research Foundation, Danone Research, Nestle, ThermoFisher Scientific, DST Diagnostic, Hycor, DBV, Aimmune, Meda Pharma, ALK, Bausch \& Lomb, Novartis, Unilever, Allergopharma, HAL, and MedUpdate.

Copyright (c) 2017 by European Society for Pediatric Gastroenterology, Hepatology, and Nutrition and North American Society for Pediatric Gastroenterology, Hepatology, and Nutrition

DOI: 10.1097/MPG.0000000000001853 
TABLE 1. Nutritional characteristics of the formulas

\begin{tabular}{|c|c|c|c|c|}
\hline & \multicolumn{2}{|c|}{ Extensive hydrolysate } & \multicolumn{2}{|c|}{ Partial hydrolysate } \\
\hline & $\mathrm{eHF}^{*}$ & Lpehf + Syn $^{*}$ & LPpHF $^{*}$ & $\mathrm{LPpHF}+\mathrm{Syn}^{*}$ \\
\hline Energy (kcal/100 mL) & 67 & 67 & 67 & 67 \\
\hline Protein & 2.3 & 1.9 & 1.9 & 1.9 \\
\hline Degree of hydrolysis & $23-29 \%$ & & $16-22 \%$ & \\
\hline \multicolumn{5}{|l|}{ Peptide size distribution (weight $\%$ ) } \\
\hline & \multicolumn{2}{|c|}{$>6.000 \mathrm{Da}:<0.1$} & \multicolumn{2}{|c|}{ >20.000 Da: max. 2} \\
\hline & \multicolumn{2}{|c|}{$3.500-6.000 \mathrm{Da}: \sim 0.4$} & \multicolumn{2}{|c|}{ 5.000-20.000 Da: $\max .9$} \\
\hline & \multicolumn{2}{|c|}{$1.500-3.500 \mathrm{Da}: \sim 12.7$} & \multicolumn{2}{|c|}{$1.000-5.000$ Da: $\max 46$} \\
\hline & \multicolumn{2}{|c|}{$<1.500 \mathrm{Da}: \sim 86.8$} & \multicolumn{2}{|c|}{$<1.000$ Da: $\max .56$} \\
\hline Carbohydrates & 10.6 & 10.7 & 11.1 & 10.7 \\
\hline Fat & 5.4 & 5.4 & 5.4 & 5.4 \\
\hline GOS $(g / 100 \mathrm{~mL})$ & - & 0.3 & - & 0.3 \\
\hline Lactobacillus fermentum $(\mathrm{cfu} / \mathrm{g})$ at production & - & $10^{7}$ & - & $10^{7}$ \\
\hline \multicolumn{5}{|l|}{ Amino acids $(\mathrm{mg} / 100 \mathrm{kcal})$} \\
\hline Alanine & 122 & 97 & 113 & 112 \\
\hline Arginine & 52 & 65 & 74 & 75 \\
\hline Asparagic acid & 290 & 221 & 220 & 218 \\
\hline Cysteine & 54 & 39 & 37 & 37 \\
\hline Glutamic acid & 488 & 371 & 317 & 316 \\
\hline Glycine & 44 & 34 & 39 & 39 \\
\hline Histidine & 45 & 45 & 49 & 48 \\
\hline Isoleucine & 148 & 117 & 113 & 111 \\
\hline Leucine & 214 & 188 & 232 & 230 \\
\hline Lysine & 239 & 174 & 216 & 214 \\
\hline Methionine & 44 & 33 & 36 & 36 \\
\hline Phenylalanine & 69 & 96 & 107 & 106 \\
\hline Proline & 176 & 132 & 97 & 98 \\
\hline Serine & 127 & 100 & 73 & 71 \\
\hline Threonine & 191 & 150 & 96 & 95 \\
\hline Tryptophan & 36 & 35 & 36 & 36 \\
\hline Tyrosine & 97 & 67 & 55 & 56 \\
\hline Valine & 132 & 107 & 94 & 94 \\
\hline
\end{tabular}

Values are expressed as g/100 kcal unless otherwise indicated. All values refer to raw materials. Due to technological processes and differences in raw material, values might not exactly reflect reference values given in correspondent directive. Formulas were manufactured and provided by HiPP GmbH \& Co. Vertrieb KG (Pfaffenhofen, Germany). AA = amino acids; $\mathrm{Da}=$ Dalton; eHf = extensively hydrolyzed protein formula, with a protein content of $2.3 \mathrm{~g}$ / $100 \mathrm{kcal} ; \mathrm{GOS}=$ galacto-oligosaccharides; $\mathrm{LP}=$ low protein; $\mathrm{LPeHF}+\mathrm{Syn}=1$ lower protein content $(1.9 \mathrm{~g} / 100 \mathrm{kcal})$, extensively hydrolyzed formula with synbiotics; $\mathrm{LPpHF}=\mathrm{LPpHF}=$ lower protein content $(1.9 \mathrm{~g} / 100 \mathrm{kcal})$, partially hydrolyzed formula.

*Individual AA were added according to Annex V of 2006/141/EC as follows: Phenylalanine and Histidine were added to both LPpHF; Phenylalanine, Tyrosine, and Tryptophan were added to both eHF and in addition Leucine and Histidine to LPeHF + Syn; Arginine was added to all LP formulas to achieve concentrations similar to those present in human milk.

\section{Randomization and Blinding}

If parents decided not to exclusively breast-feed, despite the recommendation, infants were randomized up to day 27 of life into 1 of 4 formula arms (Fig. 1) by a computer-generated list. Randomized infants were stratified by sex and family risk of allergy [assessed by questionnaire (21)]. Mothers were advised to feed intervention formula as the only substitute to human milk until the child reached the age of 16 weeks.

\section{Study Procedure}

During the baseline visit, standardized interviews were conducted in order to obtain core data (infant, maternal, parental, and "allergic" home environment) as recommended by European Society for Paediatric Gastroenterology Hepatology and Nutrition (ESPGHAN) $(22,23)$.

Parents were asked to complete a daily diary starting at Visit 1 and a 3-day record before each visit including information on stool characteristics and digestion history, formula intake, frequency of breast milk intake, and others. In case of a serious adverse event (SAE), feeding of study formula was stopped whenever recommended by a physician. Further details on the study procedure are provided in the Supplemental Content and Supplemental Figure 1 (Supplemental Digital Content, http:// links.lww.com/MPG/B201).

\section{Sample Size}

Sample size requirements were calculated using PASS 2006 (NCSS, Kaysville, Utah). Using a one-sided two-sample $t$-test procedure to show noninferiority and assuming a true difference of $-0.75 \mathrm{~g}$ in daily weight gain (pooled SD of $5.71 \mathrm{~g}$ ) with a noninferiority-margin of $-3.5 \mathrm{~g} / \mathrm{day}$ (type 1 error of $2.5 \%$, type 2 error of 20\%) resulted in 69 infants per group. Parameters for sample size estimation were derived from Ziegler et al (8). The noninferiority margin assumed a weight difference between groups of $294 \mathrm{~g}$. On the basis of this trial, the lower limit of difference in 


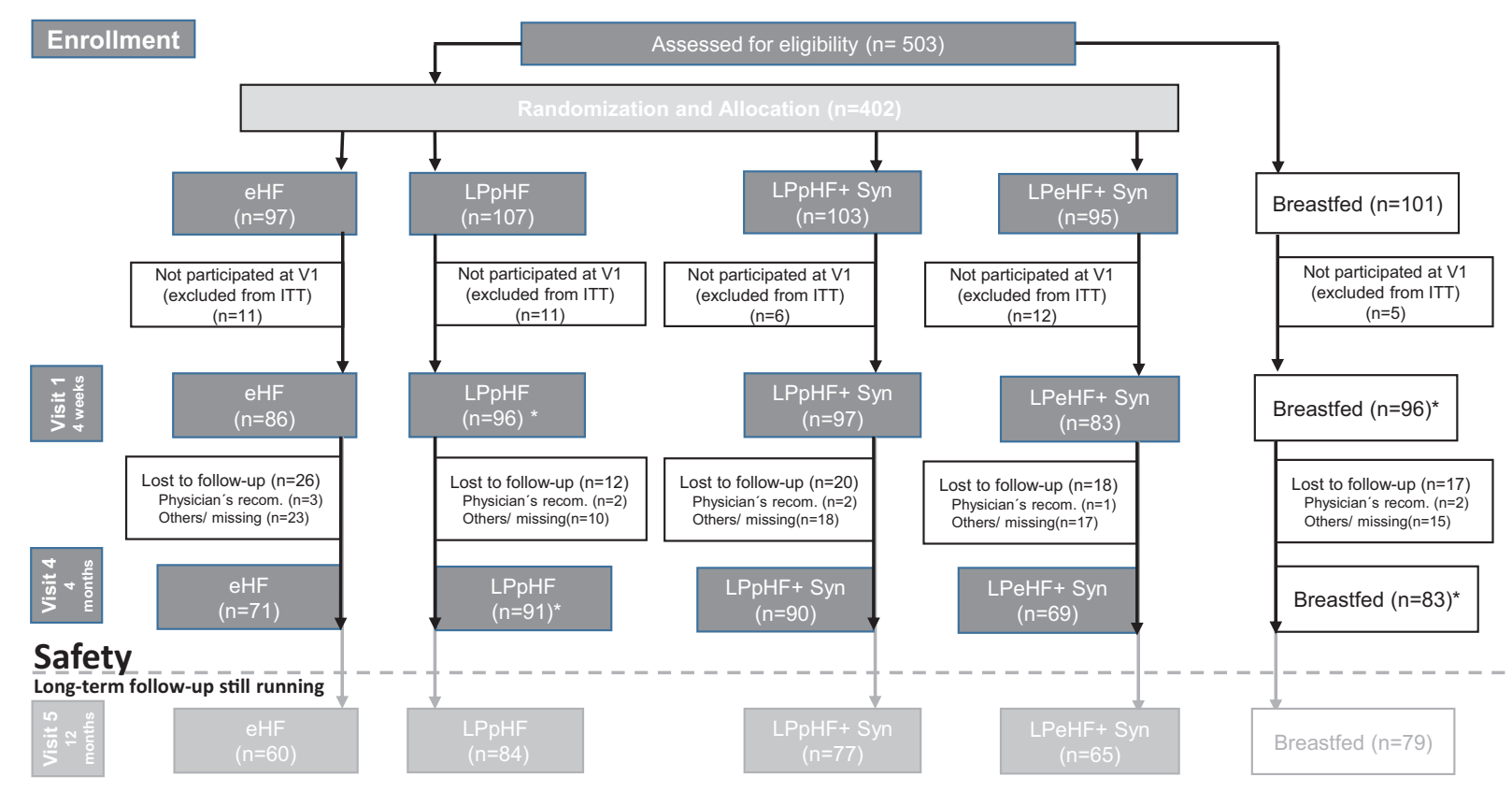

* Of which $\mathrm{n}=1$ infant in LPpHF and $\mathrm{n}=1$ infant in Breastfed group was excluded from ITT analysis due to subsequently detected failure to satisfy major entry criteria.

FIGURE 1. Subject disposition/randomization, allocation, and follow-up of study participants in the intervention group. The final ITT population included 361 formula-fed infants (Belgrade: $n=97$; Berlin: $n=89$; Bochum: $n=40$; Rostock: $n=52$; and Vienna: $n=83$ ). Of these, a total of 226 infants fulfill the criteria for the PP population (eHF: $\mathrm{n}=45$; LPpHF: $\mathrm{n}=65$; LPpHF + Syn: $\mathrm{n}=59$; LPeHF: $\mathrm{n}=57$ ). Of the 95 breast-fed infants, 66 infants fulfill criteria for PP population. eHF= extensively hydrolyzed protein formula, with a protein content of $2.3 \mathrm{~g} / 100 \mathrm{kcal}$; ITT =intention to treat population; $\mathrm{PP}=$ per protocol; $\mathrm{LPeHF}+\mathrm{Syn}=$ lower protein content $(1.9 \mathrm{~g} / 100 \mathrm{kcal})$, extensively hydrolyzed formula with synbiotics; $\mathrm{LPpHF}=$ lower protein content $(1.9 \mathrm{~g} / 100 \mathrm{kcal})$, partially hydrolyzed formula.

weight gain of $-3.5 \mathrm{~g} /$ day is clinically relevant. Assuming a dropout rate of $30 \%$, the total number of infants per group needed for recruitment was 100 .

\section{Statistical Analysis}

The ITT population comprised all enrolled infants that participated at least in Visit 1, including those with minor and severe noncompliance to protocol and subjects with missing values. The PP analysis included all infants who completed the intervention period up to 4 months of age without severe noncompliance to the protocol. Main conclusions on the primary efficacy parameter were based on the PP population, intention to treat (ITT) population served as sensitivity analysis.

Anthropometric measurements had been assessed with descriptive statistical methods and expressed as $z$ scores relative to the growth standards of the World Health Organization (WHO) for children (24). An analysis of variance model was applied to evaluate the noninferiority of the primary outcome (using fixed factors treatment group, center, allergy risk class, and sex), and additionally executed for the comparison of the other formula groups to the standard formula. One-sided $97.5 \%$ confidence limits were derived to decide on noninferiority (Data analysis: SAS version 9.3 (SAS Institute Inc., Cary, North Carolina).

Details for AA data analysis, calculations on energetic efficiency for growth (25) and further evaluation of group differences are shown in the Supplemental Content (Supplemental Digital Content, http://links.lww.com/MPG/B201).

\section{RESULTS}

\section{Recruitment and Study Population}

Between April 2010 and November 2013, 503 healthy fullterm newborns were recruited in Germany (Berlin, Bochum, Rostock), Austria (Vienna), and Serbia (Belgrade). Of these, 402 infants were randomized to four formula-feeding groups (Fig. 1). The number of subjects $(n=69)$ needed to reach statistical power was achieved for each group. One hundred and one infants were breast-fed and served as external reference (Fig. 1). The final ITT population included 361 formula-fed infants. Of these, a total of 226 infants fulfilled the criteria for the PP population.

\section{Baseline Characteristics}

Baseline characteristics did not differ between the groups except for education, smoking behavior, and age at enrollment; indicating more mothers smoked during pregnancy in the LPpHF group and none in the $\mathrm{BF}$ group $(P<0.05)$, infants in the $\mathrm{LPeHF}+\mathrm{Syn}$ group were older at enrollment $(P<0.05)$ and mothers of BF group had higher education $(P<0.001$; Supplemental Table 1, http://links.lww.com/MPG/B201).

\section{Weight Gain and Growth}

Daily weight gain of infants receiving hydrolyzed formulas with LP content did not differ from infants receiving the regular protein formula eHF (Fig. 2; Supplemental Figure 2, http:// links.lww.com/MPG/B201). The difference in daily weight gain 


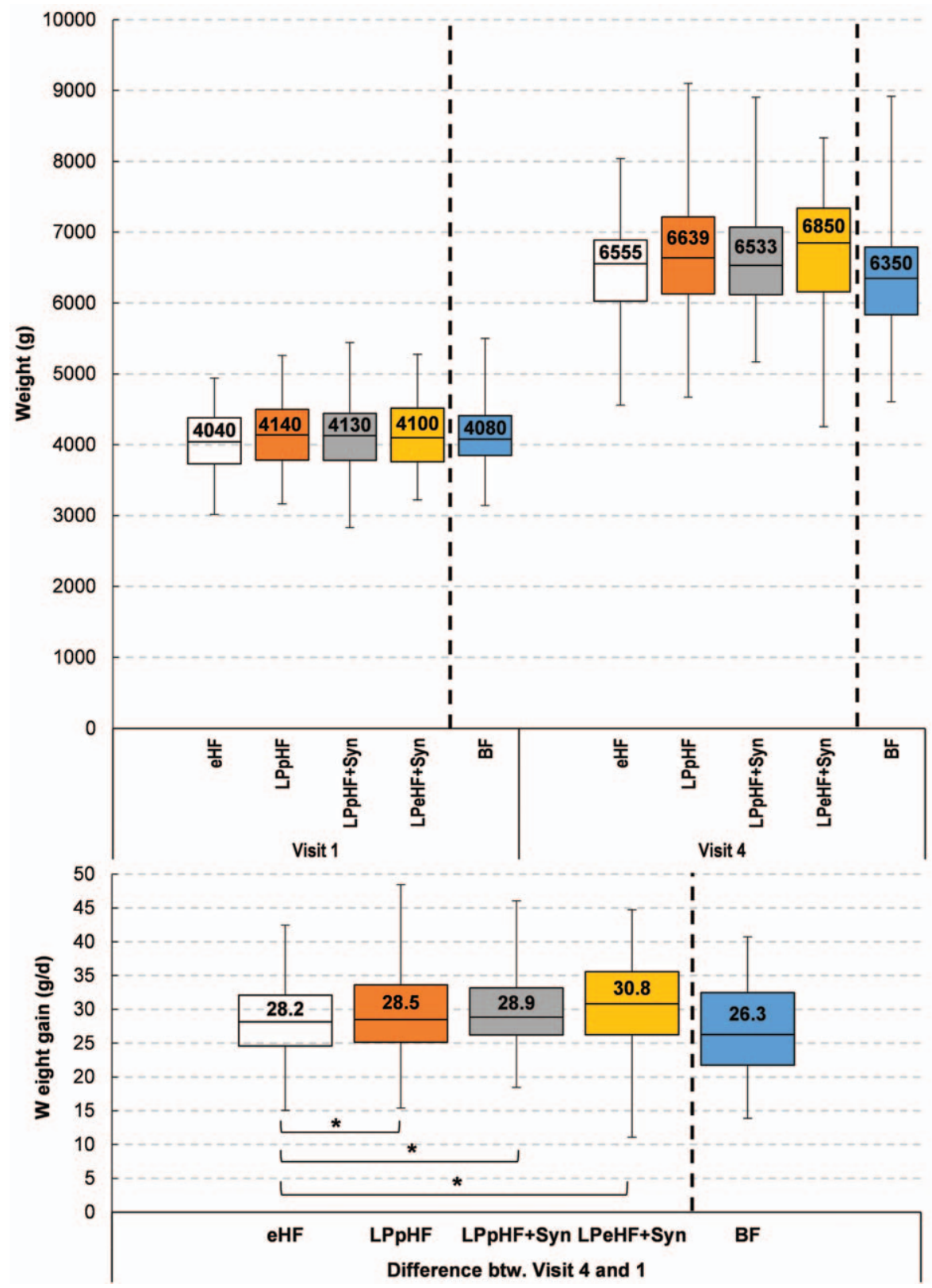

FIGURE 2. Visualization of the median absolute weight (inter quartile range, minimum and maximum; upper display) and average daily weight gain of infants between Visit 1 (day $28 \pm 3$ ) and Visit 4 (day 112 \pm 3 ) receiving modified intervention formulas compared to the standard formula (lower display). The BF group served as an external reference (ITT population). The symbol ( ${ }^{*}$ ) shows proof of noninferiority. BF $=$ breast-feeding; $\mathrm{ITT}=$ intention to treat.

in infants receiving LPpHF compared with infants fed with regular eHF was $2.15 \mathrm{~g} /$ day (CI -0.18 to inf.) for PP and $1.03 \mathrm{~g} /$ day (CI -1.01 to inf.) for ITT. The noninferiority was proven by confirming that the difference in weight gain did not exceed $3.5 \mathrm{~g}$ /day (limit of the one-sided $97.5 \% \mathrm{CI}$ ). Furthermore noninferiority was shown for both LP hydrolyzed formulas containing synbiotics (LPpHF + Syn $1.23 \mathrm{~g} /$ day (CI -0.95 to inf.), LPeHF + Syn $2.10 \mathrm{~g} /$ day (CI -0.30 to inf.) for PP. Analyses for ITT resulted in comparable results (LPpHF $1.03 \mathrm{~g} /$ day (CI -1.01 to inf.), $\mathrm{LPpHF}+\mathrm{Syn} 1.03 \mathrm{~g} /$ day (CI -0.68 to inf.), $\mathrm{LPeHF}+\operatorname{Syn} 2.25 \mathrm{~g} /$ day (CI -0.01 to inf.). ANCOVA sensitivity analysis indicated a gender effect for all group comparisons (to control).
Between Visit 1 and Visit 4, gains in weight, length and head circumference did not differ between low- and regular protein formula groups (Supplemental Table 2, http://links.lww.com/ $M P G / B 201)$. Anthropometric measurements, expressed as $z$ scores (Fig. 3), were within -1 to 1 (15th/85th percentile) throughout the study, confirming age-appropriate development.

With regard to formula ( $\mathrm{mL} /$ day) and caloric intake $(\mathrm{kcal} /$ day), no differences were found between low- and regular protein groups. As expected, however, protein intake was significantly lower in all LP groups than the eHF group $(P<0.001$; Supplemental Table 3, http://links.lww.com/MPG/B201). No differences in intakes of liquids or weaning food were observed. 


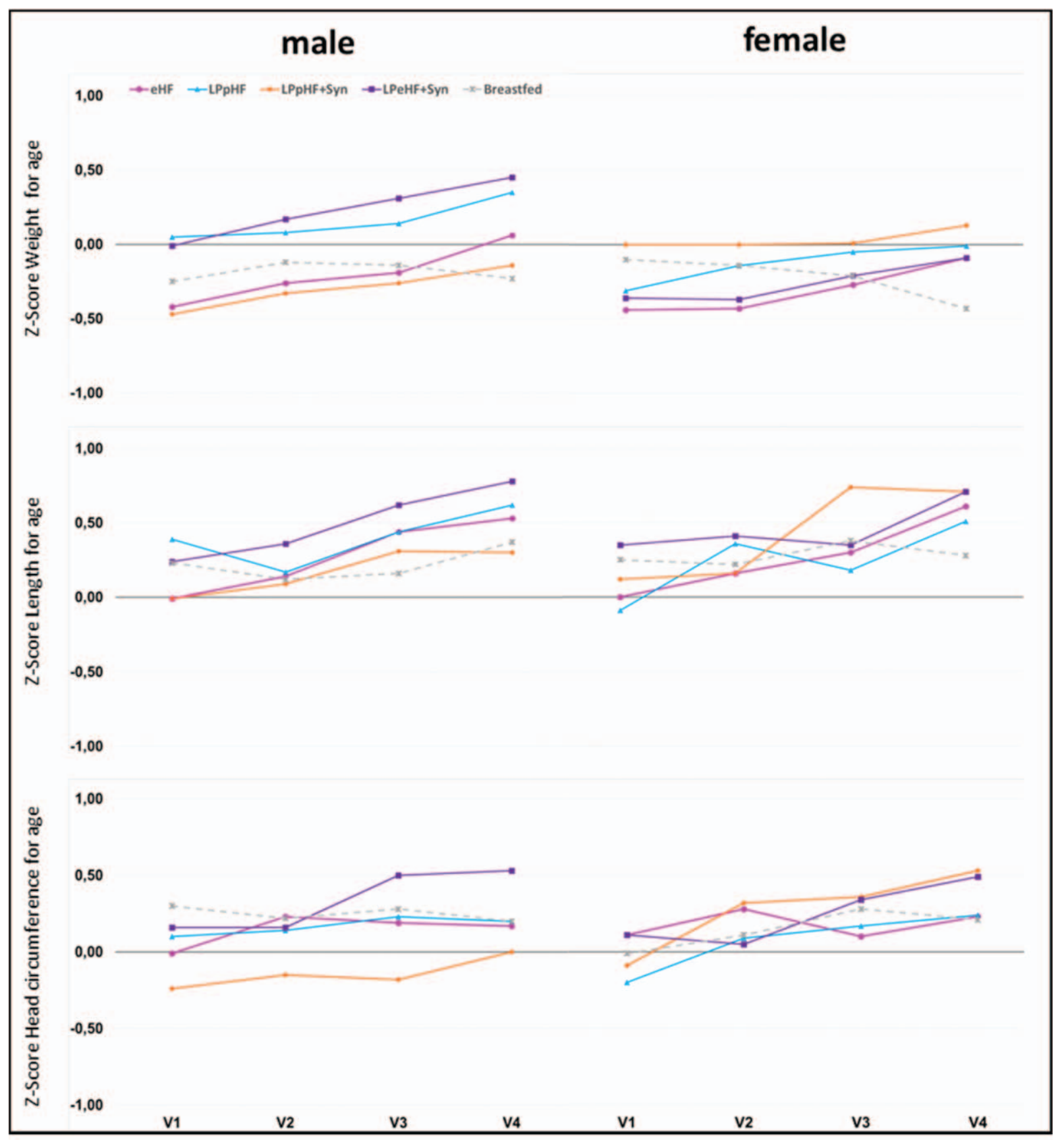

Data represent the ITT population.

FIGURE 3. Anthropometric measurements (weight for age, length for age, and head circumference for age) expressed as $z$ scores (growth standards of the WHO). $z$ scores within -1 to 1 confirm an age-appropriate development ( $z$ score $= \pm 1$ equal to 15 th/ 85 th percentile). Results of the total ITT population are depicted. ITT = intention to treat; $\mathrm{WHO}=$ World Health Organization.

The energetic efficiency for growth (weight) adjusted for center showed no difference between eHF $(5.51 \pm 1.23 \mathrm{~g} /$ $100 \mathrm{kcal})$ and LPpHF $(5.64 \pm 1.31 ; P=0.53)$ or LPpHF + Syn $(5.63 \pm 1.13 ; \quad P=0.54)$ but was higher in LPeHF + Syn $(6.12 \pm 2.02)$ compared with eHF $(P=0.03)$. No statistical difference was observed between LPpHF and LPpHF + Syn $(P=0.92)$, whereas the energetic efficiency for growth was higher in LPeHF + Syn compared with $\mathrm{LPpHF}+\mathrm{Syn}(P=0.045)$ or $\operatorname{LPpHF}(P=0.056)$.

\section{Safety Parameters}

Values for base excess $(0 \pm 3 \mathrm{mmol})$, serum bicarbonate ( $\sim 24 \mathrm{mmol} / \mathrm{L}$ ) and plasma albumin (Visit 1: $25-46 \mathrm{~g} / \mathrm{L}$; Visit 4: 
$38-54 \mathrm{~g} / \mathrm{L}$ ) were within normal range and did not differ between all formula-fed groups. Infants who fed an intervention formula had lower blood urea nitrogen values $(P<0.001)$ compared with controls and were within normal range $(6-25 \mathrm{mg} / \mathrm{dL}$; Supplemental Table 4, http://links.lww.com/MPG/B201).

Analysis of plasma AA profile focused on plasma levels at Visit 4, because at this time study, formula was the sole or predominant source of nutrition (according to study protocol PP versus ITT analysis).

At Visit 4, significantly lower plasma concentrations of Isoleucine (Ile), Methionine (Met), Proline (Pro), Threonine (Thr), Valine (Val) were observed in all intervention groups compared with eHF (Supplemental Table 5, http://links.lww.com/ $M P G / B 201)$, reflecting lower AA intake, and they approached levels observed in BF infants. Concentrations of Pro and Val were disproportionately decreased and even below the BF levels. Lysine (Lys) concentration were lower in the LPeHF + Syn group compared with eHF, also reflecting LP, and hence AA intakes. Plasma Phenylalanine (Phe) concentrations were significantly increased in all intervention groups compared with eHF.

For safety evaluation, stool characteristics were documented and differed significantly $(P<0.001$ for consistency/color/odor) between groups. The majority of infant stools in the formula groups were described as "soft." Stools of BF infants were mostly described as "watery." At Visit 4, stool color of infants receiving eHF, was described as "yellow" (60\%). "Green" was the predominant color for all LP groups (48-60\%). On study completion, the majority of parents (formula and BF group) described stool odor as normal $(83-87 \%)$ with the other parents describing the odor as "smelly" or "mixed." No significant differences in stool characteristic were observed between infants receiving formulas with or without the addition of synbiotics. Finally, no differences were reported concerning reflux and vomiting.

Results of documented AEs showed no formula related risk (Supplemental Table 6, http://links.lww.com/MPG/B201).

In total, at least one SAE (in all cases hospitalizations) have been recorded for 47 children. Documented SAEs were unlikely $(15.3 \%)$ and not related $(84.7 \%)$ to formula intake (Supplemental Table 7, http://links.lww.com/MPG/B201).

\section{DISCUSSION}

\section{Main Findings and Comparison With Other Studies}

The results of this prospective, multicenter, randomized intervention trial confirmed the hypothesis that healthy term infants consuming either partially or extensively hydrolyzed formula with LP-with or without the addition of synbioticsshowed similar growth (noninferior growth) when compared with infants fed a regular protein formula containing an extensive hydrolysate. Findings are in agreement with the only other study to date supporting normal growth in term infants receiving a formula containing a lower concentrated partial protein hydrolysate $(1.9 \mathrm{~g} / 100 \mathrm{kcal})(8)$. Furthermore, studies using intact protein have documented normal growth in infants receiving formula with a reduced protein/energy ratio $(1.8 \mathrm{~g} / 100 \mathrm{kcal})(26-28)$. Likewise, a normal weight gain of "protein-hydrolysate formula-fed infants" was shown in the study by Mennella et al (29) but the weight gain was accelerated in cow-milk formula-fed infants, although both formulas were isocaloric and the protein hydrolysate had a $35 \%$ higher protein content. The authors discussed this observation especially upon underlying hypothesis that relative to intact proteins, hydrolyzed proteins are absorbed and metabolized differently (29).
Thus, our results demonstrated that all 3 formulas with reduced contents of hydrolyzed protein, provide adequate growth of healthy infants.

Simple comparison of the LPpHF versus LPpHF + Syn groups, provide no indication that the addition of synbiotics may have influenced infants' growth, which is in line with other studies $(31,32)$. In addition, the effect of supplemented synbiotics especially on allergy development will be evaluated in the future when the follow-up beyond 4 months of age has been completed.

Importantly, all 3 interventional formula groups showed safety parameters similar to the regular formula group.

Analyzed metabolic parameters were within the reference ranges, reflecting a balanced composition of the tested formulas. The importance of the evaluation of formulas with regard to their capacity to provide normal nutritional status has not least been underlined by Hernell and Lönnerdal (32) who performed a nutritional evaluation of protein hydrolysate formulas (with no reduced protein content) in healthy term infants: plasma AAs, hematology, and trace elements. They concluded that "A reduced and more balanced amino acid content of hydrolysate formulas may be beneficial.' As expected, in our study, plasma urea nitrogen values were lower in infants receiving LP formulas, due to the reduction in protein intake (32). Notably, plasma urea nitrogen concentration did not fall to values of inadequate protein intake (33).

The significant LP intake in infants receiving LP formula resulted, at least in part, in lower plasma AA concentrations, supporting data from previous studies $(34,35)$ and this appears desirable as the plasma levels are nearer to those found in $\mathrm{BF}$ infants. The exception was Phe, with elevated plasma concentrations in infants receiving intervention formulas compared with controls. Phe was added to all intervention formulas to meet EU Guidelines for Tyr + Phe (2006/141/EC). Thus, the higher Phe levels in infant's blood plasma may just reflect formula composition. Our results, however, suggest that the European guidelinebased amount of Phe added to infant formula might be further reduced while still meeting breast-fed plasma Phe concentrations. The observed Phe plasma levels were comparable with infants receiving a nonhydrolyzed LP formula (35) and below those observed in infants fed a partially hydrolyzed protein formula in previous studies (34). Lowering protein content significantly reduced plasma threonine concentration in the LPeHF + Syn, thus, improving Thr status relative to BF infants. In general, it was observed that plasma amino acid levels reflected the composition of the different formulas. Ile, Met, Pro, Thr, and Val, were not only significantly lower in all 3 different LP groups but also lower in the LP formulas compared with eHF. Although Pro and Val levels were even below plasma levels in BF infants, the median values were similar to blood levels found in infants in previous studies who were breast-fed or received nonhydrolyzed LP formula $(25,35,36)$. As all formula groups showed a similar amino acid pattern, it is worth mentioning that aromatic AAs and branched-chain amino acids (BCAA), which have been associated with obesity in childhood or adulthood studies (37-39), behaved differently.

The LP content in intervention formulas was not compensated for by an increased intake of formula, other liquids, or complementary foods. Similar observations are described in other publications focusing on LP infant formulas (5). Energetic efficiency was comparable with that found in other studies in this age group $(25,40)$. Differences between LP groups, however, might be related to slightly lower energy intake by LPeHF + Syn.

Infants' weight, length, and head circumference were comparable between intervention and control groups (in PP and ITT population). 


\section{Study Strength and Limitations}

This study tests different hydrolysates as part of a LP formula. Major strengths of this study were subject randomization, sample size, and its multicenter, multinational design. Furthermore, major potential confounding factors were considered in the study design and standardized anthropometric measurements were performed. Small age differences at time of enrollment had no influence on study outcomes, as age was the same in all groups at study start.

As hydrolyzed formulas differ by the degree of hydrolysis and residual antigenicity, hydrolyzation alone does not guarantee clinical safety and effectiveness. Consequently, each hydrolysate needs to be tested in a clinical trial for its safety and efficacy before use (9). A study limitation is, that only one type of control formula with eHF has been analyzed. Nevertheless, results of this trial indicate safety and suitability of two individual hydrolysates (extensive and partial) tested.

\section{CONCLUSIONS}

In summary, this randomized intervention trial documents that all tested lower protein hydrolyzed formulas with or without synbiotics show noninferiority with regard to growth compared with a regular protein extensively hydrolyzed formula in infants during the first 4 months of life. Likewise, metabolic safety parameters and plasma AA concentrations reflect a safe and balanced nutritional composition.

Acknowledgments: We thank all the families who participated in the study and the medical and nursing staff at the participating hospitals, especially we thank: Henning Johannsen, Johanna Bellach, Ingrid Lawnitzak, Susanne Paschke-Goossens (Charité Universitätsmedizin Berlin); Prof Dr Uwe Schauer, PD Dr Anjona Schmidt-Choudhoury, Sandra Böger (Ruhr University Bochum); Dr Volker Schmidt, Simone Baumgärtner (Klinikum Südstadt Rostock); Dr Christoph Binder, Dr Andreas Repa, Dr Margarita Thanhäuser, Alexandra Kreißl (Medical university of Vienna); Dr Ljiljana Sipka, Dr Branka B. Trisic, Dr Snezana Zdjelar, Nina Lukic (Clinical hospital center and HiPP study center Belgrade).

\section{REFERENCES}

1. Koletzko B, Chourdakis M, Grote V, et al. Regulation of early human growth: impact on long-term health. Ann Nutr Metab 2014;65:101-9.

2. Ong KK, Loos RJF. Rapid infancy weight gain and subsequent obesity: systematic reviews and hopeful suggestions. Acta Paediatr 2006; 95:904-8.

3. Toschke AM, Grote V, Koletzko B, et al. Identifying children at high risk for over- weight at school entry by weight gain during the first 2 years. Arch Pediatr Adolesc Med 2004;158:449-52.

4. Von Kries R, Koletzko B, Sauerwald T, et al. Breast feeding and obesity: cross sectional study. BMJ 1999;319:147-50.

5. Koletzko B, von Kries R, Closa R, et al. Lower protein in infant formula is associated with lower weight up to age $2 \mathrm{y}$ : a randomized clinical trial. Am J Clin Nutr 2009;89:1836-45.

6. Koletzko B, Broekaert I, Demmelmair H, et al. Protein intake in the first year of life: a risk factor for later obesity? The EU Childhood Obesity project. In: Koletzko B, Dodds PF, Akerblom H, Ashwell M, eds. Early Nutrition and Its Later Consequences: New Opportunities. Dordrecht, Netherlands: Kluwer Academic; 2005:69-79.

7. Patro-Golab B, Zalewski BM, Kouwenhoven SM, et al. Protein concentration in milk formula, growth and later risk of obesity: a systematic review. J Nutr 2016;146:551-64.

8. Ziegler EE, Jeter JM, Drulis JM, et al. Formula with reduced content of improved, partially hydrolyzed protein and probiotics: infant growth and health. Monatsschr Kinderheilkd 2003;151:S65-71.
9. Commission Delegated Regulation (EU) 2016/127 of 25 September 2015 supplementing Regulation (EU) No. 609/2013 of the European Parliament and of the Council as regards the specific compositional and information requirements for infant formula and follow-on formula and as regards requirements on information relating to infant and young child feeding. OJEU 2016;59:1-30.

10. Fleischer DM, Spergel JM, Assa'ad AH, et al. Primary prevention of allergic disease through nutritional interventions. J Allergy Clin Immunol Pract 2013;1:29-36.

11. Muraro A, Halken S, Arshad SH, et al., EAACI Food Allergy and Anaphylaxis Guidelines Group. EAACI food allergy and anaphylaxis guidelines. Primary prevention of food allergy. Allergy 2014;69:590601.

12. NIAID-Sponsored Expert PanelBoyce JA, Assa'ad A, et al. . Guidelines for the diagnosis and management of food allergy in the United States: report of the NIAID-Sponsored Expert Panel. J Allergy Clin Immunol 2010;126:S1-58.

13. Høst A, Koletzko B, Dreborg S, et al. Dietary products used in infants for treatment and prevention of food allergy. Joint Statement of the European Society for Paediatric Allergology and Clinical Immunology (ESPACI) Committee on Hypoallergenic Formulas and the European Society for Paediatric Gastroenterology, Hepatology and Nutrition (ESPGHAN) Committee on Nutrition. Arch Dis Child 1999;81:80-4.

14. Von Berg A. The role of hydrolysates for allergy prevention-pro. Pediatr Allergy Immunol 2013;24:720-3.

15. Bode L. The functional biology of human milk oligosaccharides. Early Hum Dev 2015;91:619-22.

16. Elazab N, Mendy A, Gasana J, et al. Probiotic administration in early life, atopy, and asthma: a meta-analysis of clinical trials. Pediatrics 2013;132:e666-76.

17. Penders J, Gerhold K, Stobberingh EE, et al. Establishment of the intestinal microbiota and its role for atopic dermatitis in early childhood. J Allergy Clin Immunol 2013;132:601-7.

18. Moher D, Hopewell S, Schulz KF, et al. CONSORT 2010 explanation and elaboration: updated guidelines for reporting parallel group randomised trials. J Clin Epidemiol 2010;63:e1-37.

19. Commission Directive 2006/141/EC of 22 December 2006 on infant formulae and follow-on formulae and amending Directive 1999/21/EC p. L 401/2, ANNEX VI.

20. Gil-Campos M, Lopez MA, Rodriguez-Benitez MV, et al. Lactobacillus fermentum CECT 5716 is safe and well tolerated in infants of 1-6 months of age: a randomized controlled trial. Pharmacol Res 2012;65:231-8.

21. Bergmann RL, Forster J, Schulz J, et al. Atopic family history. Validation of instruments in a multicentre cohort study. Pediatr Allergy Immunol 1993;4:130-5.

22. Aggett P, Agostoni C, Axelsson I, et al. Core data for nutrition trials in infants: a discussion document-a commentary by the ESPGHAN Committee on Nutrition. J Pediatric Gastroenterol Nutr 2003;36: $338-42$.

23. Koletzko B, Fewtrell R, Gibson, et al. Consensus Group on Outcome Measures Made in Paediatric Enteral Nutrition Clinical; Early Nutrition Project. Core data necessary for reporting clinical trials on nutrition in infancy. Ann Nutr Metab 2015;66:31-5.

24. WHO Multicenter Growth Reference Study Group. WHO Child Growth Standards: length/height-for-age, weight-for-age, weightfor-length, weight-for-height and body mass index-for-age: methods and development. Geneva: World Health Organization; 2006. Available at: http://www.who.int/childgrowth/software/en/. Accessed March 29, 2018.

25. Fleddermann M, Demmelmair H, Grote V, et al. Infant formula composition affects energetic efficiency for growth: the BeMIM study, a randomized controlled trial. Clin Nutr 2014;33:588-95.

26. Turck D, Grillon C, Lachambre E, et al. Adequacy and safety of an infant formula with a protein/energy ratio of $1.8 \mathrm{~g} / 100 \mathrm{kcal}$ and enhanced protein efficiency for term infants during the first 4 months of life. J Pediatr Gastroenterol Nutr 2006;43:364-71.

27. Räihä NC, Fazzolari-Nesci A, Cajozzo C, et al. Whey predominant, whey modified infant formula with protein/energy ratio of $1.8 \mathrm{~g} / 100$ kcal: adequate and safe for term infants from birth to four months. J Pediatr Gastroenterol Nutr 2002;35:275-81. 
28. Abrams SA, Hawthorne KM, Pammi M. A systematic review of controlled trials of lower-protein or energy-containing infant formulas for use by healthy full-term infants. Adv Nutr 2015;6:178-88.

29. Mennella JA, Ventura AK, Beauchamp GK. Differential growth patterns among healthy infants fed protein hydrolysate or cow-milk formulas. Pediatrics 2011;127:110-8.

30. Braegger C, Chmielewska A, Decsi T, et al. Supplementation of infant formula with probiotics and/or prebiotics: a systematic review and comment by the ESPGHAN committee on nutrition. J Pediatr Gastroenterol Nutr 2011;52:238-50.

31. Burks AW, Harthoorn LF, Van Ampting MT, et al. Synbiotics-supplemented amino acid-based formula supports adequate growth in cow's milk allergic infants. Pediatr Allergy Immunol 2015;26:316-22.

32. Hernelle O, Lönnerdal B. Nutritional evaluation of protein hydrolysate formulas in healthy term infants: plasma amino acids, hematology, and trace elements. Am J Clin Nutr 2003;78:296-301.

33. Fomon SJ, Ziegler EE, Nelson SE, et al. Infant formula with proteinenergy ratio of $1.7 \mathrm{~g} / 100 \mathrm{kcal}$ is adequate but may not be safe. J Pediatr Gastroenterol Nutr 1999;28:495-501.
34. Decsi T, Veitl V, Burus I. Plasma amino acid concentrations, indexes of protein metabolism and growth in healthy, full-term infants fed partially hydrolyzed infant formula. J Pediatr Gastroenterol Nutr 1998;27:12-6.

35. Kirchberg FF, Harder U, Weber M, et al. Dietary protein intake affects amino acid and acylcarnitine metabolism in infants aged 6 months. $J$ Clin Endocrinol Metab 2015;100:149-58.

36. Karlsland Akeson PM, Axelsson IE, Räihä NC. Protein and amino acid metabolism in three- to twelve-month-old infants fed human milk or formulas with varying protein concentrations. J Pediatr Gastroenterol Nutr 1998;26:297-304.

37. Perng W, Gillman MW, Fleischer AF, et al. Metabolomic profiles and childhood obesity. Obesity (Silver Spring) 2014;22:2570-8.

38. Hellmuth C, Kirchberg FF, Lass N, et al. Tyrosine is associated with insulin resistance in longitudinal metabolomic profiling of obese children. J Diabetes Res 2016;2016:2108909.

39. Rauschert S, Uhl O, Koletzko B, et al. Metabolomic biomarkers for obesity in humans: a short review. Ann Nutr Metab 2014;64:314-24.

40. Fleddermann M, Demmelmair H, Koletzko B. Energetic efficiency of infant formulae: a review. Ann Nutr Metab 2014;64:276-83. 